\title{
EFFECTS OF MIXTURE INHOMOGENEITY ON THE AUTO-IGNITION OF REACTANTS UNDER HCCI ENVIRONMENT
}

\author{
Ramanan Sankaran*and Hong G. $\operatorname{Im}^{\dagger}$ \\ Department of Mechanical Engineering \\ University of Michigan, Ann Arbor, MI 48109
}

\begin{abstract}
As an attempt at providing insight to develop better modeling strategies for HCCI engines, the ignition and propagation of a reaction front in a premixed fuel/air stream mixed with hotter exhaust gases is computationally investigated using the opposed-flow configuration. The effects of heat and radical transport are studied by imposing various mixing rates on the system. The results show that the scalar dissipation rate and mixture inhomogeneities have a significant effect on auto-ignition of the local mixture. Two different modes of front propagation are identified; the spontaneous propagation mode and the diffusion controlled mode. In the former case, mixing slows down ignition due to loss of radicals from the ignition kernel, while in the latter the transport of radicals and heat is mainly responsible for propagation of the front. A criterion to distinguish the two ignition modes is suggested based on the characteristic time scales of the auto-ignition and diffusion. The results show that lower mixing rate and higher pressure in general favors ignition in the spontaneous propagation mode. A parametric mapping of the ignition delay as a function of the mixture fraction and scalar dissipation rate suggests a simplified modeling strategy in multi-dimensional simulation of HCCI engines.
\end{abstract}

\section{INTRODUCTION}

The homogenous charge compression ignition (HCCI) engines have recently emerged as a viable choice for a next-generation internal combustion engine. In an HCCI engine, fuel and air are well mixed and induced into the engine cylinder. This charge is then compressed to a high pressure and temperature which ignites the mixture without the need for an external ignition system. The HCCI engine shows a potential to combine the advantages of both SI and CI engines, in that the auto-ignition of reactants allows higher efficiencies, while the lean homogeneous mixture can substantially reduce the NOx and soot formation.

\footnotetext{
* Student Member AIAA, Graduate Student

${ }^{\dagger}$ Assistant Professor
}

To understand HCCI ignition and to provide predictive tools to identify the engine design parameters, efforts have been made to model HCCI engine combustion with various levels of complexity. Modeling approaches range from a simple zero-dimensional formulation which assumes that fluid motion or mixture inhomogeneities do not affect auto-ignition ${ }^{1-3}$ to full 3-dimensional CFD simulations coupled with detailed chemistry ${ }^{4,5}$. As a compromise between these two, a multi-zone modeling approach ${ }^{6}$ has also been applied to account for interactions between adjacent zones, such as heat transfer and work, while simplifying the detailed flow field and effect of turbulence on chemical reactions. These wide range of studies have suggested that a zero-dimensional model does not properly predict the start of ignition as it does not account for mixture inhomogeneities. It is important to characterize the ignition event under an environment similar to $\mathrm{HCCI}$ engine.

One of the main challenges towards the development of the HCCI engine is to control the auto-ignition process and to achieve smooth energy release during the cycle. To accomplish these goals, various techniques have been suggested as a means to control the ignition and combustion processes. Among them, exhaust gas recirculation (EGR) has been found to be an effective means to reduce engine knock and to extend the operating range of the engine ${ }^{7,8}$. There is evidence that mixture preparation, inhomogeneity and turbulence can significantly affect ignition delay and burn duration ${ }^{9-11}$. It is anticipated that these factors can be exploited to achieve the desired operating conditions.

With exhaust gas recirculation, fresh reactants are mixed with exhaust gases which are at a higher temperature but are depleted in reactants. This leads to inhomogeneities in temperature and mixture composition, and formation of certain hot spots or kernels which are initially more reactive than the rest of the mixture. Chemical reactions are initiated in this hot spot and later propagate into the remainder of the mixture in the form of a reaction front. Zeldovich ${ }^{12}$ has identified various regimes that characterize a reaction front propagating through a non-uniform mixture, such as (a) a supersonic deflagration wave (b) a detonation wave (c) a subsonic deflagra- 
tion wave and (d) a molecular and thermal diffusion controlled flame front. In the HCCI application, smooth engine operation requires the combustion to take place in either of the last two modes only. In this paper, the subsonic deflagration wave will be called the spontaneous propagation wave, thereby distinguishing it from the diffusion controlled flame front mode. Bradley et al. ${ }^{13,14}$ and Chen et al. ${ }^{15}$ have examined these modes of propagation numerically and observed the presence of a spontaneous propagation mode with velocities much larger than the conventional diffusion controlled flame. However, the relative importance of these two modes and the effect of fluid mechanical strain is yet to be understood.

Accounting for the effects of inhomogeneities and studying the propagation of auto-ignition under different mixing rates will improve our understanding of the fundamental characteristics of HCCI combustion. This will provide a rational and physically-based means to predict start of combustion in the engine cycle, which is crucial to the overall performance of the engine. The main goal of the present study is to systematically investigate these effects and provide insights towards more accurate modeling strategies in the HCCI application. In particular, we perform a parametric study by imposing different mixing rates on a reactive mixture with temperature gradient and study the ignition time and subsequent front propagation.

\section{PROBLEM CONFIGURATION}

In practical HCCI engines, temperature inhomogeneities occur due to various causes, such as insufficient mixing with residual exhausts, wall heat transfer, and variation in compression heating due to differences in the specific heat ratio, $\gamma=C_{p} / C_{v}$. However, the primary source for temperature inhomogeneities is the mixing with residual exhaust gases. Numerical simulations by Babajimopoulos et al. ${ }^{16}$ demonstrated that there exists a very strong correlation between temperature and mixture composition within an HCCI engine during the intake and compression stages. Their results also show that the reactions first initiate at the hotter spots which have lower amounts of fuel and then propagate into the colder fuel-rich mixtures.

The main objective of this study is to investigate the ignition and propagation of the reaction front when a fresh fuel/air stream is allowed to mix with a hotter exhaust stream at different mixing rates. The opposedflow configuration allows an extensive parametric study of different conditions at low computational cost. With fresh fuel/air mixture flowing through one nozzle and burnt products through the other, various mixing rates can be imposed by varying the velocity of flow through the nozzles. This study can be readily extended to different types of mixing, such as mixing between two reactant

\begin{tabular}{c|c|c|c}
\hline Case & $\mathrm{P}(\mathrm{atm})$ & $\mathrm{T}_{\text {fuel }} \mathrm{K}$ & $\mathrm{T}_{\text {exhaust }} \mathrm{K}$ \\
\hline 1 & 40 & 800 & 1100 \\
2 & 80 & 900 & 1200 \\
\hline
\end{tabular}

Table 1: Pressure and temperature initial conditions.

mixtures with different composition, by imposing appropriate boundary conditions.

The governing equations for the opposed flow configuration and details of the numerical implementation can be found in Ref. ${ }^{17}$. The steady solutions are obtained using a modified version of OPPDIF ${ }^{18}$ and the unsteady solutions using OPUS ${ }^{19}$. These codes are interfaced with Chemkin ${ }^{20}$ and Transport ${ }^{21}$ for computing the reaction rates and transport properties.

Methane has been chosen as the fuel for this study, to avoid the complexity of higher hydrocarbons and also because natural gas continues to be a candidate for HCCI engines. The full methane-air kinetic mechanism (GRI $3.0)^{22}$ has been used. Premixed methane/air mixture at an equivalence ratio of $\phi=0.3$ flows through one nozzle against another stream of the corresponding products of complete combustion. A low pressure and a high pressure case, corresponding to pressures at top dead center of a naturally aspirated and supercharged engine are considered to account for differences in mixture reactivity. The pressure and temperatures at the nozzle are given in table 1 .

\section{RESULTS AND DISCUSSION}

For the conditions discussed above, the level of mixing of the two streams can be characterized by a mixture fraction variable, $Z$. If the fresh $\mathrm{CH}_{4} / \mathrm{Air}$ and burnt products are mixed in the ratio, $Z:(1-Z)$, the resultant mixture composition is,

$$
Y_{k}=Z Y_{k, f}+(1-Z) Y_{k, e}
$$

where $Y_{k}$ is mass fraction of species $k$ and subscripts $f$ and $e$ denote the fuel and exhaust streams respectively. Similary, temperature of the mixture can be determined by adiabatically mixing the enthalpies of the two streams as,

$$
h=Z h_{f}+(1-Z) h_{e} .
$$

Therefore, any given value of $Z$ uniquely determines the composition and the enthalpy of the mixture. As a reference case, the auto-ignition delay for the perfectlyhomogeneous mixture corresponding to various $Z$ was calculated by a zero-dimensional model using SENKIN ${ }^{23}$. The results for Case 1 are shown in Fig. 1. Here the ignition delay $\left(\tau_{i, 0}\right)$ is defined as the time at which $5 \%$ of the total heat release is generated. It is seen that the location of shortest ignition delay is close to $Z=0$, where 


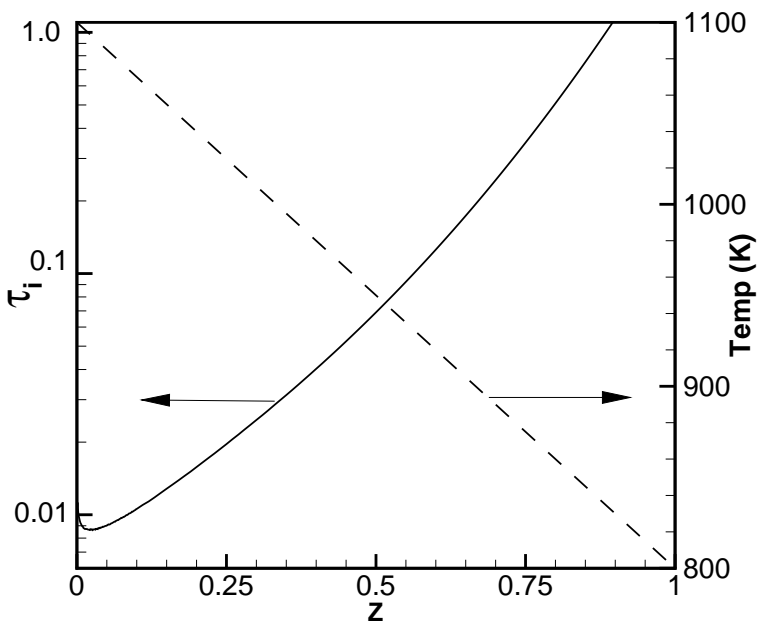

Figure 1: Mixture temperature and homogenous autoignition delays as a function of $\mathrm{Z}$ for Case 1 .

the fuel concentration is very low but the temperature is high.

If we ignore the influence of adjacent mixture and assume that different points in the $Z$ field ignite purely based on their local initial conditions, each location in a mixture field would then ignite at its respective 0-D auto-ignition time. By comparing the results of the counterflow system with these reference conditions, we can systematically assess and quantify the additional effects resulting from transport and mixing that occur in actual combustion systems.

\section{Steady Ignition Limit}

For the counterflow cases described in the previous section, we determine the steady ignition limit by successively computing steady solutions at reduced nozzle velocities until the ignition turning point is reached. Figure 2 shows a typical response of peak temperature and $\mathrm{H}$ mole fraction as the velocity at the nozzle is decreased. For Case 1, the ignition turning point occurs at $V=26.6 \mathrm{~cm} / \mathrm{s}$. For velocities above this limit, the reaction is suppressed due to insufficient residence time, and thus a "frozen" steady solution is obtained. With the nozzle velocity below this limit, however, the system becomes "ignitable" and we can compute the unsteady transition of the system from a frozen initial state to the final reacting state as the ignition progresses. In order to obtain an initial frozen solution, the steady state nonreacting flow field is determined by artificially suppressing the reaction rates in the simulation. Then to initiate ignition, the chemical reactions are turned on.

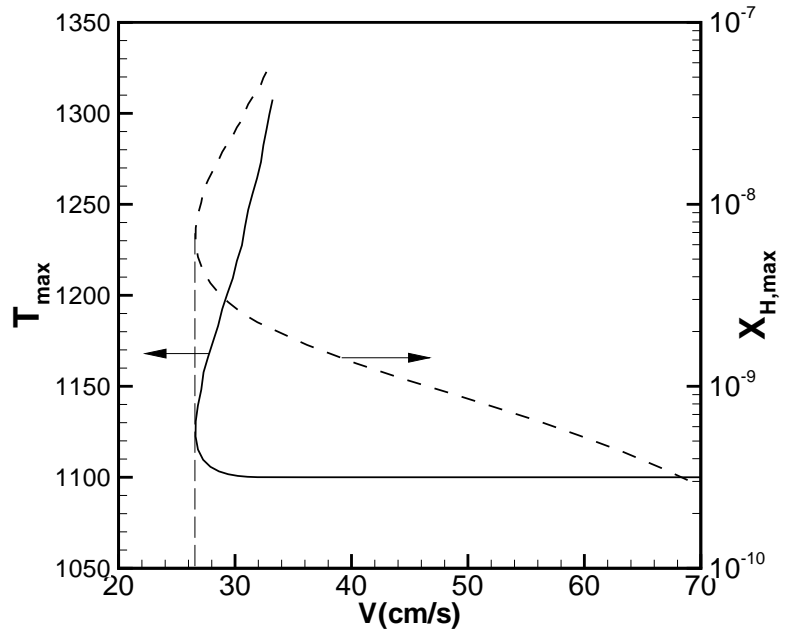

Figure 2: Steady ignition turning point behavior of the premixed $\mathrm{CH}_{4}$ /air stream for Case 1 .

Effects of Mixing Rate on Ignition

Figure 3 shows evolution of temperature profile for Case 1. For the solution shown, velocity at the nozzle inlet was $5 \mathrm{~cm} / \mathrm{s}$. The mixture fraction, $Z$, is determined at every point in the domain using equation (2). Figure 4 shows the temperature evolution in the mixture fraction space. It is seen that the auto-ignition initiates at a point closer to the hot exhaust stream and then develops into a reaction front which progresses towards the fuel-rich region. The time history of a mixture at a specific value of $Z$ is monitored as it undergoes ignition under the influences of transport processes and the ignition delay is computed. As in the homogeneous case, the ignition delay $\left(\tau_{i}\right)$ is defined as the time at which $5 \%$ of the total heat release is produced. When the velocity at the nozzle is increased, gradients in the system steepen, thereby increasing the dissipation rates of heat and radicals.

Figure 5 shows the ignition delay $\left(\tau_{i}\right)$ versus $Z$ for Case 1 at various nozzle velocities. Also shown are the reference 0-D auto-ignition delays from Fig. 1 for comparison. First, it is noted that the 0 -D auto-ignition delays are shorter than the $1-\mathrm{D}$ results for values of $Z \lesssim 0.2$ and longer for larger values of $Z$. A second observation is that there is a crossing of lines at values of $Z$ around 0.5 , which implies that the strain rate can have a non-monotonic effect on the ignition delay. These results show that the effect of diffusional transport on ignition can either delay or advance the ignition event, depending on initial reactivity of the mixture. For mixtures with very short $\tau_{i, 0}$ (for small $Z$ ), the presence of transport processes, as in our counterflow configuration, leads to loss of heat and radicals from the ignition kernel to 


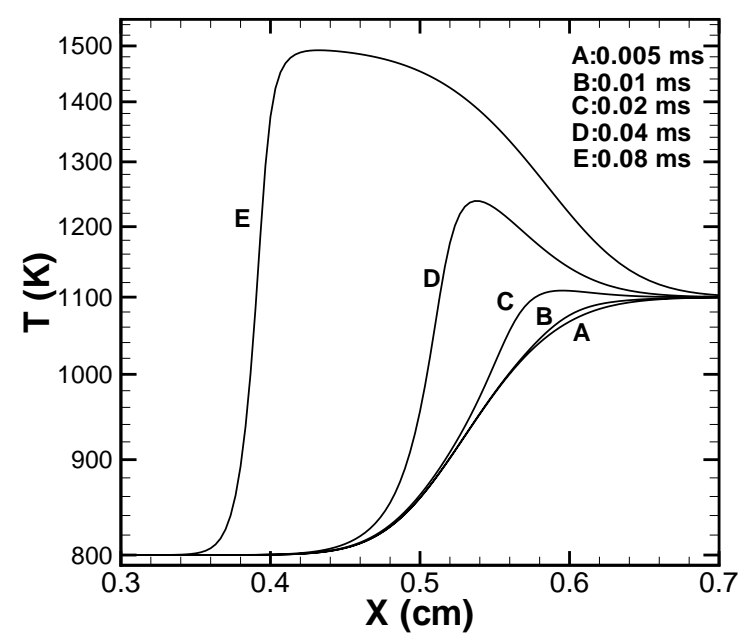

Figure 3: Evolution of temperature profiles from the frozen state as ignition progresses for $V=5 \mathrm{~cm} / \mathrm{s}$.

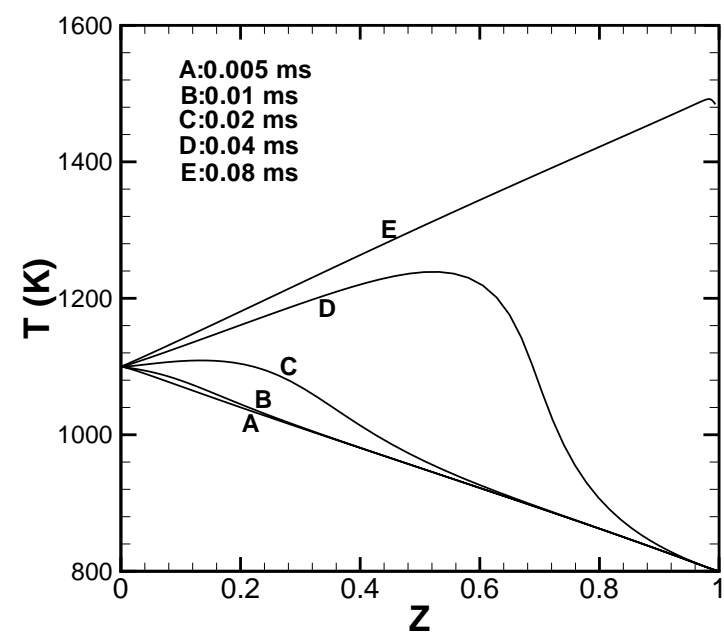

Figure 4: Evolution of temperature profiles in the mixture fraction space for the case shown in fig. 3.

the surroundings, thereby further delaying the ignition compared to the homogenous counterpart. When $\tau_{i, 0}$ is very long (for large $Z$ ), however, the diffusion of heat and radicals from the adjacent reacting zone facilitates ignition. Therefore, the mechanism of ignition for the regimes can be clearly distinguished: the former is primarily spontaneous propagation of the chemically-driven reaction front, while the latter is a front propagation that is assisted by the diffusion. The transport effect serves as a loss in the former, and as a crucial component of ignition in the latter.

The solution procedure is repeated for Case 2 at

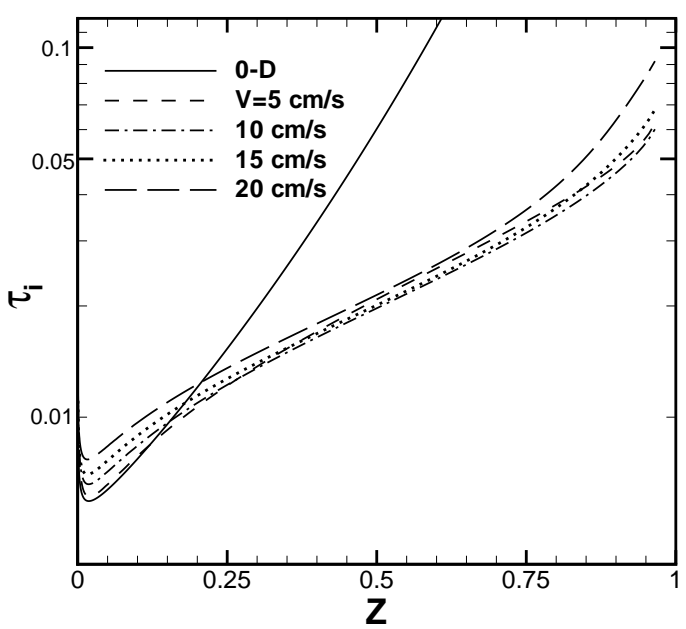

Figure 5: Ignition delay as a function of $Z$ for Case 1 at various nozzle velocities.

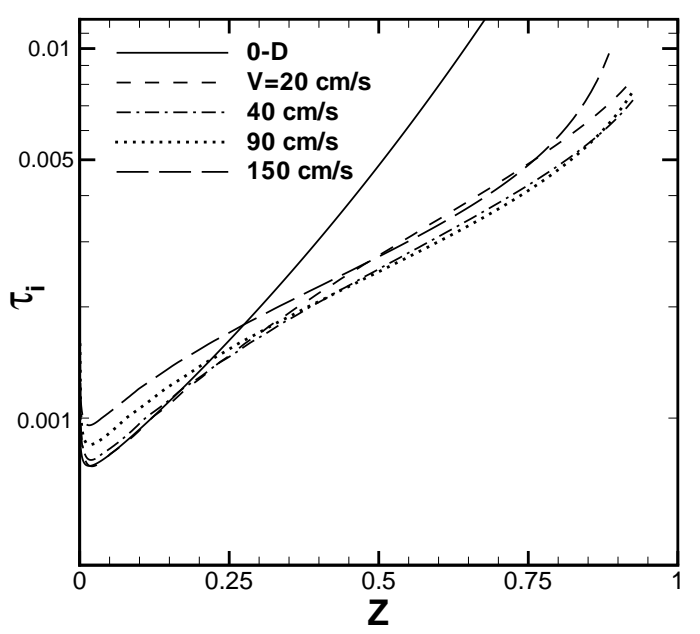

Figure 6: Ignition time as a function of $Z$ for Case 2 at various nozzle velocities.

a higher range of nozzle velocities and the results are shown in Fig. 6. In this case, due to the higher pressure and temperatures the reactivity of the mixture is higher and the ignition delays are much shorter, in the order of milliseconds. Nevertheless, qualitatively similar behavior is observed in terms of the ignition delay versus the mixing rate.

\section{Front Propagation Speed}

It is also of interest to identify the propagation speed of the reaction front. Due to the presence of temperature gradient, adjacent points in space ignite at different in- 


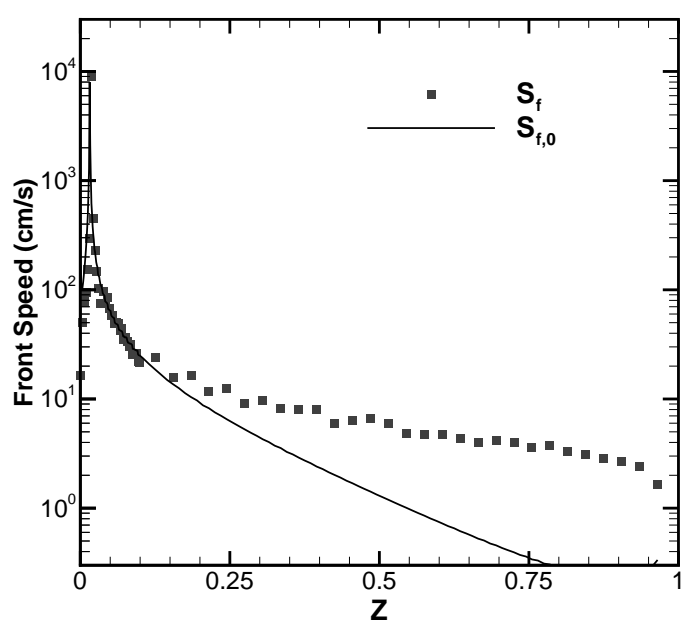

Figure 7: Reaction front speed $S_{f}$ (symbols) and $S_{f, 0}$ (line) as a function of $Z$ for Case 2 at $V=20 \mathrm{~cm} / \mathrm{s}$.

stants, either with or without the support of diffusion, and thereby generate an ignition wave that propagates through the mixture. Knowing the ignition time $\tau_{i}$ at each spatial location $x$, the propagation speed of the front can be defined as

$$
S_{f}=\left(\frac{d \tau_{i}}{d x}\right)^{-1}=\left(\frac{d \tau_{i}}{d Z} \cdot \frac{d Z}{d x}\right)^{-1} .
$$

Therefore, for the unsteady counterflow solutions $S_{f}$ can be determined either directly by evaluating $d \tau_{i} / d x$ in the physical space or by $d \tau_{i} / d Z$ in the mixture fraction space with the knowledge of $Z(x)$. Likewise, the corresponding 0 -D front propagation speed can be determined by

$$
S_{f, 0}=\left(\frac{d \tau_{i, 0}}{d Z} \cdot \frac{d Z}{d x}\right)^{-1} .
$$

In other words, $S_{f, 0}$ represents the hypothetical front propagation speed for the same $Z(x)$ distribution without the mixing effects. The difference between $S_{f}$ and $S_{f, 0}$ indicates the effect of mixing and transport on ignition, which needs to be accounted for in the multi-dimensional modeling.

Figure 7 shows the results of comparison between the two propagation speeds for Case 2 at a nozzle velocity of $20 \mathrm{~cm} / \mathrm{s}$. While there is good agreement for lower values of $Z$ where spontaneous propagation is dominant, the differences are substantial for higher values of $Z$, where the effects of mixing in facilitating the wave propagation is clearly shown.

\section{Reaction Front Regimes}

Thus far, the characteristics of the two distinct ignition modes have been discussed. We now want to iden- tify an appropriate criterion to distinguish the two modes. This can be done by comparing the characteristic time scales of diffusion and spontaneous propagation. First, we define a characteristic length scale, $\Delta x$, based on flow strain rate, $\kappa$, and thermal diffusivity, $\alpha$, as

$$
\Delta x=\left(\frac{\alpha}{\kappa}\right)^{1 / 2} .
$$

This length scale in the physical space is converted to $\Delta Z$ in the mixture fraction space using the relation

$$
\Delta Z=\Delta x \cdot \frac{d Z}{d x},
$$

or

$$
\Delta Z=\left(\frac{\chi}{2 \kappa}\right)^{1 / 2}
$$

where $\chi$ is the scalar dissipation rate defined as,

$$
\chi=2 \alpha\left(\frac{d Z}{d x}\right)^{2}
$$

Therefore, the time scale of diffusion process across the length scale $\Delta Z$ in mixture fraction space is given by

$$
\tau_{d}=\frac{\Delta Z^{2}}{\chi}
$$

On the other hand, the time taken for the spontaneous propagation wave to traverse the same distance is

$$
\tau_{s p}=\frac{d \tau_{i, 0}}{d Z} \cdot \Delta Z
$$

The ratio of these two time scales is defined as the transition parameter, $\beta$ :

$$
\beta=\frac{\tau_{s p}}{\tau_{d}} .
$$

For $\beta<1$, the time scale of the diffusion processes are longer and the ignition is in the spontaneous propagation mode. On the other hand, for $\beta>1$, the time scale of diffusion is shorter and the ignition occurs as a diffusion-controlled wave propagation. The parameter $\beta$ can therefore be used as a criterion to demarcate the two regimes. Figures 8 and 9 show the computed values of $\beta$ for Cases 1 and 2 , and the predicted transition point based on the criterion $\beta=1$. It is noted that, when the gradients in the mixing layer are steepened due to an increase in the nozzle velocity, the fraction of mixture igniting in the diffusion-controlled propagation mode is increased. Furthermore, compared to Case 1, a larger portion of the mixture in Case 2 burns in spontaneous mode. This shows that an initial condition of higher reactivity favors spontaneous propagation.

Incidentally, it should be noted that the present analysis considers a constant pressure system where the effects of compression heating are neglected. In a constant 


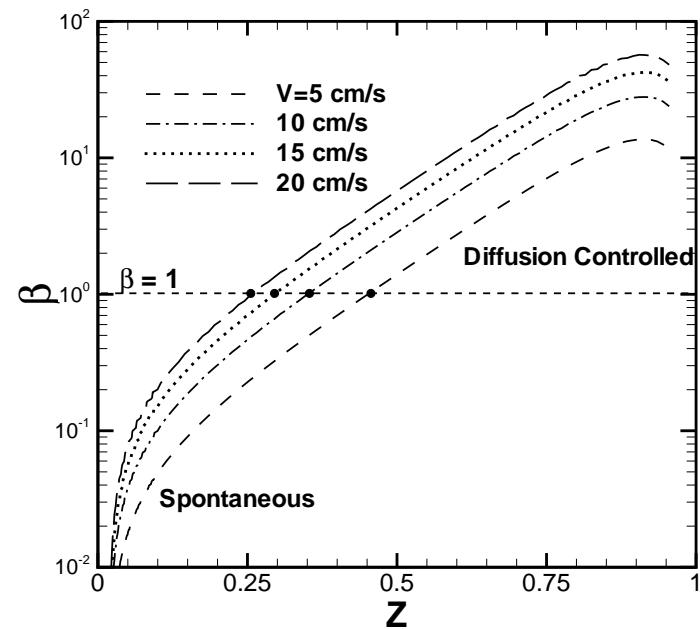

Figure 8: Transition parameter, $\beta$, as a function of the mixture fraction for various velocities, for Case 1.

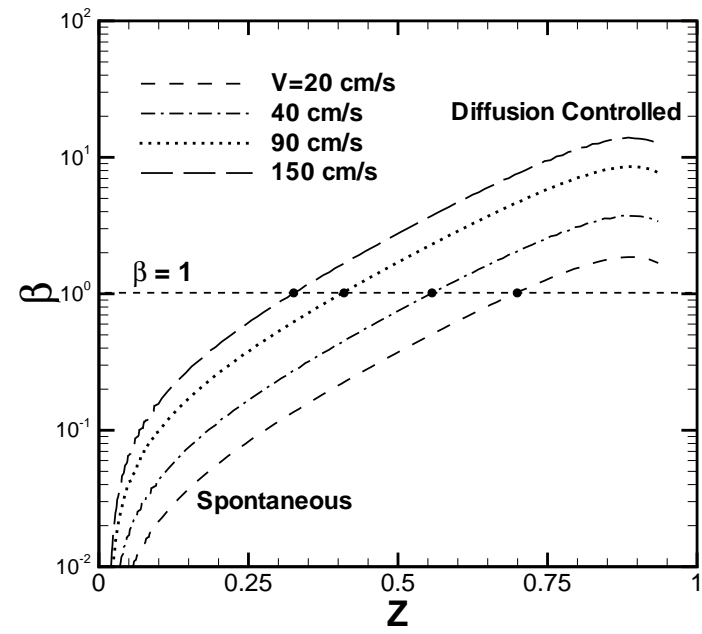

Figure 9: Transition parameter, $\beta$, as a function of the mixture fraction for various velocities, for Case 2 .

volume system, the heat released by the early combustion will raise the pressure and heat up the end gas due to compression. Consequently, the predicted ignition delay may be slightly longer than the actual engine combustion cases, although such effect is expected to be small as far as the determination of ignition is concerned. Nevertheless, the results clearly demonstrate that the local dissipation due to inhomogeneity can have a significant effect on premixed auto-ignition, similar to the non-premixed ignition results.

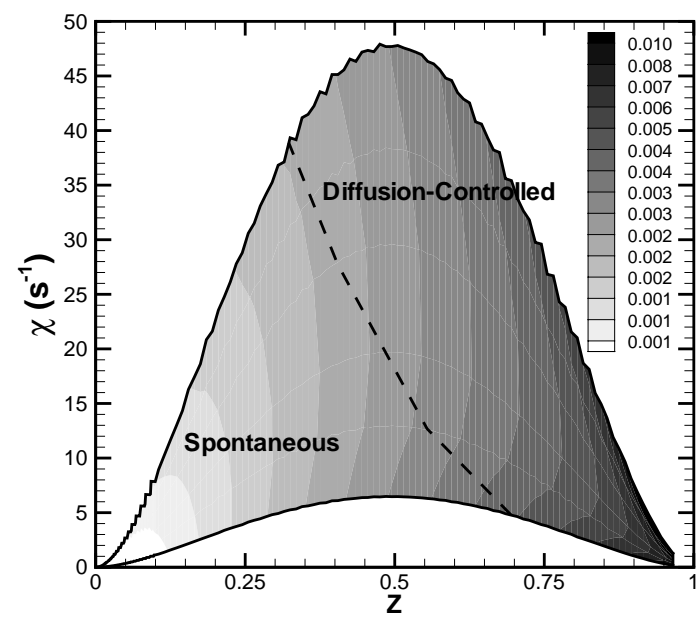

Figure 10: Mapping of ignition delay time (in s) as a function of the mixture fraction and scalar dissipation rate for Case 2.

\section{Parameterization of Mixing Effects}

In the previous sections, it was found that the ignition delay of a mixture between methane-air and exhaust gases depends primarily on two physical parameters, namely the mixture fraction $(Z)$, which represents the degree of mixing, and the scalar dissipation rate $(\chi)$, which is a measure of the rate of mixing. Therefore, a mapping of the ignition delay in terms of these two parameters can provide a framework to develop physical submodels to predict ignition in large-scale simulations. A multi-dimensional CFD code can compute the mean mixture fraction and local scalar dissipation rate of different spatial locations in the cylinder. Since ignition is a highly local phenomena, prediction of ignition based on cell-averaged quantities can lead to significant error. Precomputed results of the 1-D mixing layer solution can provide a more accurate description of ignition throughout the grid cells at considerably lower cost.

Figure 10 shows an example of the mapping for Case 2. A range of $\chi$ space can be spanned by computing a number of cases at different inlet velocities, as shown in Fig. 6. The contour plot clearly indicates the general trend that the ignition delay becomes longer as the mixture fraction increases. For a fixed mixture fraction value, a slight non-monotonic response in the ignition delay as $\chi$ increases is also shown. The dashed line is the transition between the spontaneous propagation and the diffusion-controlled propagation modes, as defined in the previous section. It is seen that the region occupied by the spontaneous propagation mode becomes narrower as the scalar dissipation rate increases. 


\section{CONCLUSIONS}

Numerical simulations were performed in an opposed flow configuration by allowing a stream of fresh premixed fuel/air mixture flow against burnt exhaust products. The effect of the mixture composition and scalar dissipation rate on the auto-ignition delay has been investigated. It is found that when the gradients in the system are steepened, the high scalar dissipation rates increase the auto-ignition delays. The results also show that the reaction front can propagate in either a spontaneous propagation or a diffusion controlled mode. A parameter to characterize the transition between these two modes was proposed by comparing their time scales. In general, it was found that lower rate of mixing and higher mixture reactivity promotes ignition in the spontaneous propagation mode. Finally, the parameterization of the ignition delay in terms of the mixture fraction and scalar dissipation rate suggested a physical submodel to describe ignition in complex multi-dimensional simulations.

\section{ACKNOWLEDGEMENT}

This work has been sponsored by the Consortium on Homogeneous Charge Compression Ignition Engine Research, directed by the University of Michigan, and funded by Department of Energy under agreement DEFC04-01AL67611.

\section{REFERENCES}

1. Smith, J. R., Aceves, S. M., Westbrook, C. K., and Pitz, W. J. In Proceedings of the 1997 ASME Internal Combustion Engine Fall Technical Conference, pp. 85-90 (1997).

2. Kelly-Zion, P. L. and Dec, J. E. Proceedings of the Combustion Institute 28:1187-1194 (2000).

3. Dec, J. E. SAE Technical Paper 2002-01-1309 (2002).

4. Agarwal, A. and Assanis, D. N. SAE Technical Paper 980136 (1998).

5. Kong, S., Marriot, C. D., Reitz, R. D., and Christensen, M. SAE Technical Paper 2001-01-1026 (2001).

6. Aceves, S. M., Flowers, D. L., Westbrook, C. K., Smith, J. R., Pitz, W., Dibble, R., Christensen, M., and Johansson, B. SAE Technical Paper 2000-010327 (2000).

7. Christensen, M. and Johansson, B. SAE Technical Paper 982454 (1998).

8. Martinez-Frias, J., Aceves, S. M., Flowers, D., Smith, J. R., and Dibble, R. SAE Technical Paper 2001-01-3613 (2001).
9. Maigaard, P., Mauss, F., and Kraft, M. ASME Paper 2000-ICE-275 (2000).

10. Christensen, M., Hultqvist, A., and Johansson, B. SAE Technical Paper 2002-01-0425 (2002).

11. Christensen, M. and Johansson, B. SAE Technical Paper 2002-01-2864 (2002).

12. Zeldovich, Y. B. Combustion and Flame 39:211214 (1980).

13. Bradley, D., Morley, C., Gu, X. J., and Emerson, D. R. SAE Technical Paper 2002-01-2868 (2002).

14. Gu, X. J., Emerson, D. R., and Bradley, D. Combustion and Flame 133:63-74 (2003).

15. Chen, J. H., Mason, S. D., and Hewson, J. C. In Proceedings of the Third Joint Meeting of U.S. Sections of the Combustion Institute (2003).

16. Babajimopoulos, A., Assanis, D. N., and Fiveland, S. B. SAE Technical Paper 2002-01-2829 (2002).

17. Im, H. G., Raja, L. L., Kee, R. J., and Petzold, L. R. Combustion Science and Technology 158:341-363 (2000).

18. Lutz, A. E., Kee, R. J., Grcar, J. F., and Rupley, F. M. OPPDIF: A Fortran Program for Computing Opposed-Flow Diffusion Flames. Tech. Rep. SAND96-8243, Sandia National Laboratories (1997).

19. Im, H. G., Raja, L. L., Kee, R. J., Lutz, A. E., and Petzold, L. R. OPUS: A Fortran Program for Unsteady Opposed-Flow Flames. Tech. Rep. SAND2000-8211, Sandia National Laboratories (2000).

20. Kee, R. J., Rupley, F. M., and Miller, J. A. ChemkinII: A Fortran Chemical Kinetics Package for the Analysis of Gas-Phase Chemical Kinetics. Tech. Rep. SAND89-8009B, Sandia National Laboratories (1991).

21. Kee, R. J., Dixon-Lewis, G., Warnatz, J., Coltrin, M. E., and Miller, J. A. A Fortran Computer Code Package for the Evaluation of Gas-Phase Multicomponent Transport Properties. Tech. Rep. SAND868246, Sandia National Laboratories (1986).

22. Smith, G. P., Golden, D. M., Frenklach, M., and et al.. http://www.me. berkeley.edu/gri_ mech/.

23. Lutz, A. E., Kee, R. J., and Miller, J. A. SENKIN: A Fortran Program for Predicting Homogeneous Gas Phase Chemical Kinetics with Sensitivity Analysis. Tech. Rep. SAND87-8248, Sandia National Laboratories (1988). 